University for Business and Technology in Kosovo

UBT Knowledge Center

UBT International Conference

2015 UBT International Conference

Nov 7th, 9:00 AM - 5:00 PM

\title{
Dual Tone Multi Frequency (DTMF) signal generation and detection using MATLAB software
}

Nihat Pamuk

Turkish Electricity Transmission Company, nihatpamuk@gmail.com

Ziynet Pamuk

Sakarya University, ziynet@sakarya.edu.tr

Follow this and additional works at: https://knowledgecenter.ubt-uni.net/conference

Part of the Computer Sciences Commons, and the Digital Communications and Networking Commons

Recommended Citation

Pamuk, Nihat and Pamuk, Ziynet, "Dual Tone Multi Frequency (DTMF) signal generation and detection using MATLAB software" (2015). UBT International Conference. 97.

https://knowledgecenter.ubt-uni.net/conference/2015/all-events/97

This Event is brought to you for free and open access by the Publication and Journals at UBT Knowledge Center. It has been accepted for inclusion in UBT International Conference by an authorized administrator of UBT Knowledge Center. For more information, please contact knowledge.center@ubt-uni.net. 


\title{
Dual Tone Multi Frequency (DTMF) signal generation and detection using MATLAB software
}

\author{
Nihat Pamuk ${ }^{1}$, Ziynet Pamuk ${ }^{2}$ \\ ${ }^{1}$ Turkish Electricity Transmission Company \\ ${ }^{2}$ Sakarya University, Electric - Electronic Engineering Department \\ nihatpamuk@gmail.com ${ }^{1}$, ziynet@sakarya.edu.tr²
}

\begin{abstract}
In this study, Dual Tone Multi Frequency (DTMF) signal generation and detection is implemented by using Goertzel Algorithm in MATLAB software. The DTMF signals are generated by using Cool Edit Pro Version 2.0 program for DTMF tone detection. The DTMF signal generation and detection algorithm are based on International Telecommunication Union (ITU) recommendations. Frequency deviation, twist, energy and time duration tests are performed on the DTMF signals. The algorithm recognizes the DTMF tones if they satisfy the recommendations, otherwise they are rejected.
\end{abstract}

Keywords: DTMF, Signal Generation and Detection, Goertzel Algorithm, Frequency Deviation

\section{Introduction}

Dual Tone Multi Frequency (DTMF) is a system of signal tones used in the field of communication whose application ranges from voice mail and help desks to telephone banking and controlling robotics designs [1]. The DTMF signal is generated by the sum of two sinusoidal tones. One of them is selected from a group of $697 \mathrm{~Hz}, 770 \mathrm{~Hz}, 852 \mathrm{~Hz}, 941 \mathrm{~Hz}$ named as low frequency group and the second one is selected from a set of $1209 \mathrm{~Hz}, 1336 \mathrm{~Hz}, 1477 \mathrm{~Hz}, 1633 \mathrm{~Hz}$ called high frequency group. By addition of two sinusoidal tones, four frequencies from each group gives a total of 16 combinations, which represented ten decimal digits, alphabet characters A, B, C, D and two special characters "*” and "\#” [2]. Table 1 shows the frequencies used for each signal.

Table 2. Frequencies used in forming DTMF tones.

\begin{tabular}{ccccc}
\hline Frequency & $1209 \mathrm{~Hz}$ & $1336 \mathrm{~Hz}$ & $1477 \mathrm{~Hz}$ & $1633 \mathrm{~Hz}$ \\
\hline $697 \mathrm{~Hz}$ & 1 & 2 & 3 & $\mathrm{~A}$ \\
$770 \mathrm{~Hz}$ & 4 & 5 & 6 & $\mathrm{~B}$ \\
$852 \mathrm{~Hz}$ & 7 & 8 & 9 & $\mathrm{C}$ \\
$941 \mathrm{~Hz}$ & $*$ & 0 & $\#$ & $\mathrm{D}$ \\
\hline
\end{tabular}

The signal tones should be detected by using Goertzel Algorithm. [3, 4, 5] have used Goertzel's algorithm for the detection of digital DTMF signals. Generating tones for all 16 digits ( 0 to $9, \mathrm{~A}$ to D, * and \#), the Cool-Edit Pro Version 2.0 program is used and the generating tones are used in MATLAB software for detection. Automatic Gain Control (AGC) is added before detection which provides the average output signal level is fed back to adjust the gain to an appropriate level for a range of input signal levels [6]. DTMF detection is implemented by using Goertzel Algorithm and tests are performed according to standards of International Telecommunication Union (ITU) by using MATLAB software.

\section{The Structure of Keypad and DTMF Signal}

DTMF is a method of representing digits with tones for communications [7]. DTMF tones are used by all touch tone phones to represent the digits on a touch tone keypad. DTMF signaling used for 
many applications such as telephone banking, routing customer support calls, system control, voicemail, and similar applications [8]. A DTMF signal represents one of sixteen touchtone symbols $(0-9, A-D, \#, *)$ as shown in figure 1 . Each symbol is represented by one of four frequencies in a low frequency band and one of four frequencies in a higher frequency band.

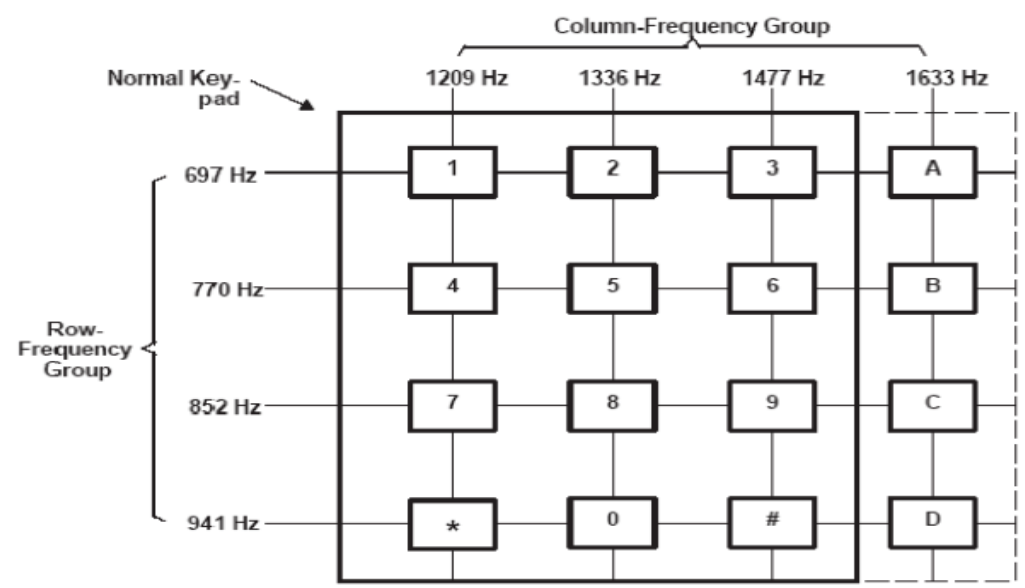

Fig. 4. Touch tone telephone keypad

In figure 1, the symbols are shown in a matrix format. The columns are represented by frequencies in a band between $1 \mathrm{kHz}$ (kilohertz) and $2 \mathrm{kHz}$, and the rows are represented by frequencies in a band between $500 \mathrm{~Hz}$ and $1 \mathrm{kHz}$. Whenever a key of a touchtone keypad is depressed, the DTMF is generated by adding two signals and transmitted to device. The device that receives this dual tone signal must detect which one of the four low frequencies and which one of the four high frequencies has been received to determine which symbol is to be determined [9].

$$
y(\mathrm{n})=A_{1} \sin \left(\frac{2 \pi \mathrm{nf}_{1}}{F_{s}}\right)+A_{2} \sin \left(\frac{2 \pi \mathrm{nf}_{2}}{F_{s}}\right)
$$

DTMF tones are generated like above equation 1 , and $\mathrm{fl}, \mathrm{f} 2$ frequencies denotes high or low frequency tone of DTMF respectively. Fs represents sampling frequency.

\section{DTMF Signal Generation and Detection}

In communications, Discrete Fourier Transform (DFT) is used for frequency detection. DFT converts the signal from time domain to frequency domain.

\subsection{Goertzel Algorithm}

Goertzel algorithm is used widely and it is most popular method in the worldwide. This algorithm uses IIR filters that are tuned to eight DTMF frequencies. Direct realization of the goertzel algorithm is showed in figure 2 . 


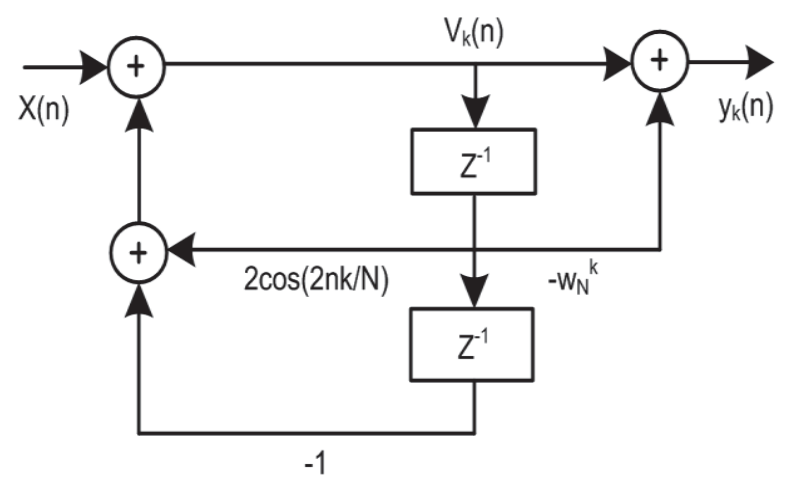

Fig. 2. Direct realization of the goertzel algorithm

Goertzel algorithm [10], can be seen from the equation 2.

$$
V_{k}(\mathrm{n})=\operatorname{coeff}(\mathrm{k}) \cdot V_{k}(\mathrm{n}-1)-V_{k}(\mathrm{n}-2)+\mathrm{x}(\mathrm{n})
$$

where Vk $(-1)=0, \mathrm{Vk}(-2)=0, \mathrm{x}(\mathrm{n})$ represents input sample. Recursively compute for $\mathrm{n}=0 \ldots \mathrm{N}$. Compute once every $\mathrm{N}$;

$$
|X(\mathrm{k})|=V_{k}^{2}(\mathrm{~N}) \cdot V_{k}^{2}(\mathrm{~N}-1)-2 \cdot \operatorname{coeff}(\mathrm{k}) \cdot V_{k}(\mathrm{~N}) \cdot V_{k}(\mathrm{~N}-1)
$$

The IIR filter coefficients for the row and column frequencies are calculated from;

$$
\operatorname{coeff}(\mathrm{k})=\cos \left(2 \cdot \pi \cdot \frac{f_{k}}{f_{s}}\right)
$$

The IIR filter coefficient for the second harmonics is found;

where $\mathrm{W}_{\mathrm{N}}^{-\mathrm{kN}}=\mathrm{e}^{-\mathrm{j} 2 \mathrm{nk}}$

$$
\text { coeff } \_2 n d(\mathrm{k})=\cos \left(2 \cdot \pi \cdot \frac{2 f_{k}}{f_{s}}\right)
$$

\subsection{Validity Checking of DTMF Signal}

Once all the row and column filter energies are calculated, a series of tests are executed to determine the validity of tone and digit results [10], [11]; signal energy, twist, relative peaks, second harmonic, duration check and frequency deviation.

Signal Energy: The DTMF detection algorithm uses adaptive threshold for the signal energy test. The signal energy varies with the input signal level. Therefore it is difficult have a fix threshold for this test. Fix threshold can be only used if the input level is same all the time. Automatic gain control can be applied to the input.

Twist: Twist is the ratio of low frequency power high frequency power. If the row tone energy is bigger than the column tone energy, this is known as normal twist. The threshold for the normal twist is $8 \mathrm{~dB}(\max )$. If the column tone energy is bigger than the row tone energy, the twist is described as reverse twist and it can be $4 \mathrm{~dB}(\max )$ [12], [13].

Relative Peaks: The energy of the strongest signal in each group (row and column tones) is compared the energies of the rest of the tones in its group. The strongest tone must stand out from the other tones in its group by a certain ratio [14].

Second Harmonic: The check of second harmonic provides whether the coming signal is DTMF or speech. Speech has second harmonics, but DTMF signals do not have second harmonics.

Duration Check: According to ITU standards, the duration of DTMF signals should be $40 \mathrm{~ms}$.

Frequency Deviation: The frequency deviation range should be between $\pm(1.5-3.5) \%$, where accepting or rejecting the DTMF tone is up to the designer. 


\section{Experimental Work and Performance Test}

In this section, first of all the DTMF tones should be generated to detect and test. After generating, we start to detect these tones by using Goertzel Algorithm. We generate the DTMF tones by using Cool Edit Pro program with different properties and characteristics. This program provides to save the signals as a wav file format which is read wavered command in MATLAB program, so the transfer is provided. The needed characteristics in Cool Edit Pro Version 2.0 are specified. The sampling rate should be $8000 \mathrm{~Hz}$ because $8000 \mathrm{~Hz}$ is used in telephony applications.

The voice of human can be approximately $3200 \mathrm{~Hz}$ and according to the Nyquist criteria the sampling rate should be twice frequency of the original signal, so $8000 \mathrm{~Hz}$ for sampling rate is enough to operate by using human voice. If the sampling rate is bigger than $8000 \mathrm{~Hz}$, it is meaningless and becomes complicated and the execution time of program is getting longer. The channel is selected mono. Stereo can be selected, but there is no need another channel for the application. The DTMF tones are generated from Cool Edit Pro program like figure 3. If we write "7" in dial string part, you will obtain this signal seen in figure 4. If we analyze the DTMF signal in frequency domain in Cool Edit Program, we get the figure 5 .

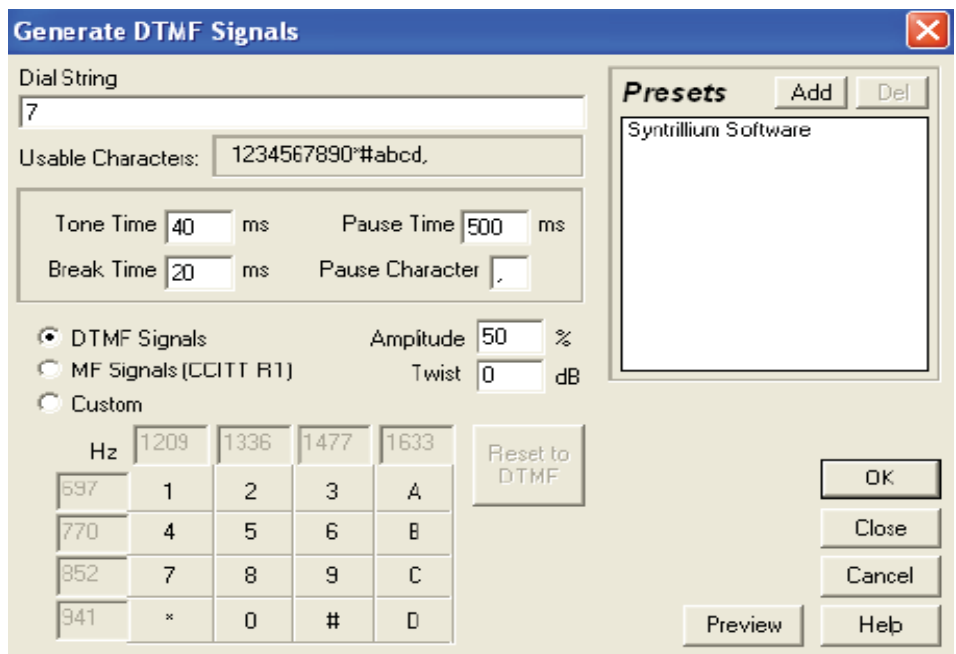

Fig. 3. The setting for dial string and time durations

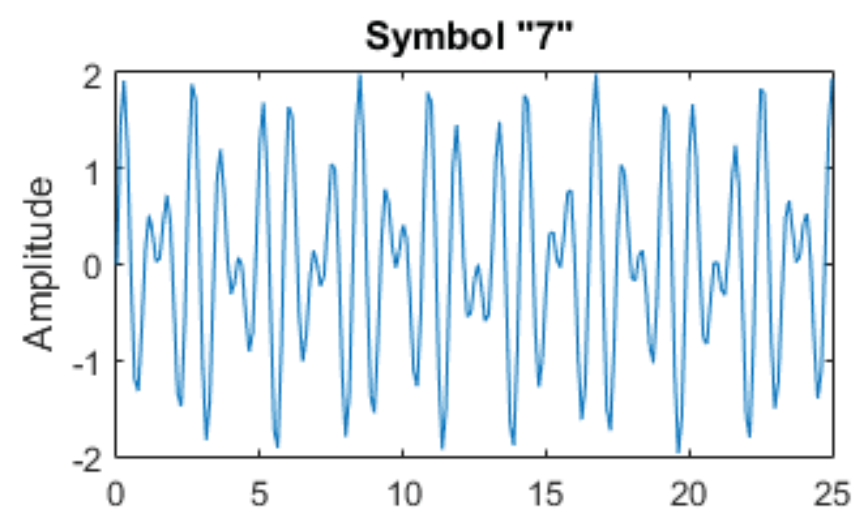

Fig. 4. The DTMF signal "7" 


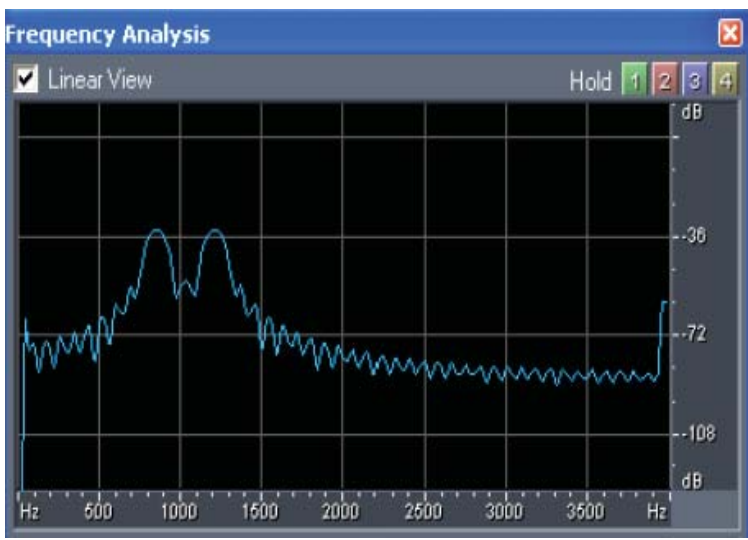

Fig. 5. The frequency analysis of the DTMF signal "7"

In order to verify my MATLAB program, we get the DFT magnitude graph in MATLAB program and saw that the program works correctly. Figure 6 shows the Goertzel DFT magnitude estimate of the DTMF signal "7" tone. As can be seen from the figure 6, DTMF "7" tone contains $852 \mathrm{~Hz}$ and $1209 \mathrm{~Hz}$ sinusoidal. After the one digit is detected by program, we generated more than one DTMF to be detected by taking buffer whose length is 128 . We generated the all DTMF tones “1234567890*\#ABCD”.

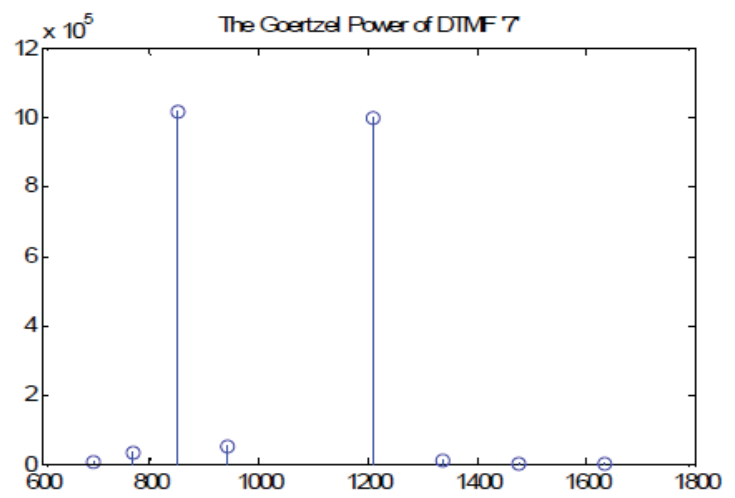

Fig. 6. The DFT magnitude of the DTMF signal "7"

After that, we performed the following test; frequency deviation, twist, duration and relative peaks. We performed the test two times to DTMF. After both of tests are satisfied, we decided whether or not the DTMF is. After the DTMF is to be recognized twice, we said that DTMF is detected correctly. The tests are performed by playing string "1234567890*\#ABCD" five times. After the tests are performed on DTMF signals five times, we detected the string "1234567890*\#ABCD". Therefore, we show that DTMF algorithm works correctly. In second harmonic test, second harmonic energy is found but second harmonic test is not performed because, we cannot talk off test tape. In the frequency deviation test, when the frequency range is between $\pm 1.5 \%$, algorithm detected correctly. When the range is selected more than $\pm 3 \%$, the algorithm failed.

\section{Conclusions}

In this study, DTMF signal detection is implemented using MATLAB 7.0 program. The DTMF signals are generated for each 16 digits in Cool Edit Pro Version 2.0 program. The signals are strengthened by adding the algorithm of AGC. Then the DTMF detection is done by using Goertzel Algorithm. For international communication some tests are to be done with the standard of ITU. First of all the detection and tests are applied for one digit, but later the consecutive DTMF digits detection 
is performed. The DTMF algorithm can be implemented in Simulink and the fixed point code can be generated from Simulink and it can be used in real time telephony applications.

\section{References}

1. Singh, A.K.: Simulation of Dual Tone Multi Frequency Detection Using Bank of Filters, International Journal of Engineering Research \& Technology (IJERT), vol. 2, no. 6 (2013) 750 755

2. Ravishankar, M.K., Hari, K.V.S.: Performance Analysis of Goertzel Algorithm Based Dual Tone Multifrequency (DTMF) Detection Schemes, Departmental Technical Report (2004)

3. Mock, P.C.: Add DTMF Generation and Decoding to DSP-mP design, EDN (1985) 205-220

4. Gay, S.L., Hartung, J., Smith, G.L.: Algorithms for Multi-Channel DTMF Detection for the WE DSP32 Family, International Conference on Acoustics Speech and Signal Processing (ICASSP) (1989) 1134-1137

5. Bagchi, S., Mitra, S.K.: An Efficient Algorithm for DTMF Decoding Using the Subband NDFT, International Symposium on Circuits and Systems, (1995) 1936-1939

6. Isaac Martinez, G.: Automatic Gain Control (AGC) Circuits Theory and Design, ECE 1352 Analog Integrated Circuits-I (Term Paper), University of Toronto (2001) 1-25

7. AN218: DTMF Decoder Reference Design Application Notes, Silicon Laboratories Inc., 4635 Boston Lane, (2005) 1-36

8. Arslan, G., Evans, B.L., Sakarya, F.A., Pino, J.L.: Performance Evaluation and Real Time Implementation of Subspace Adaptive and DFT Algorithms for Multi Tone Detection, Proc. IEEE International Conference on Telecommunications, (1996) 884-887

9. US Patent 6608896.: Efficient Digital ITU Compliant Zero Buffering DTMF Detection Using the Non-uniform Discrete Fourier Transform (2003)

10. Schmer, G.: DTMF Tone Generation and Detection: An Implementation Using the TMS320C54x, Digital Signal Processing Solutions Application Note, Texas Instruments, SPRA096A (2000) 119

11. Mock, P.: Add DTMF Generation and Decoding to DSP-P Designs, DSP Applications with the TMS320 Family, vol. 1, Texas Instruments (1989)

12. Edizkan, R.: DTMF Decoder Design for Fixed-Point DSPs (2005)

13. Emiya, V., Lucio, F.C., Vallot, P.D., Melles, D.: Generic Tone Dtection Using Teager-Kaiser Energy Operations on the Starcore SC140 core (2004)

14. Proakis, J.G., Manolakis, D.G.: DTMF Tone Generation and Detection: An Implementation Using the TMS320C54x, Digital Signal Processing Solutions Application Report, Texas Instruments, SPRA096 (1997) 1-56 